\title{
Factors Associated with Delay in Breast Cancer Presentation at the Only Oncology Center in North East Ethiopia: A Cross-Sectional Study
}

This article was published in the following Dove Press journal: Journal of Multidisciplinary Healthcare

\author{
Anissa Mohammed Hassen (iD) ' \\ Foziya Mohammed Hussien (iD) \\ Zinet Abegaz Asfaw' \\ Hussien Endris Assen ${ }^{2}$ \\ 'Department of Public Health, School of \\ Public Health, College of Medicine and \\ Health Science, Wollo University, Dessie, \\ Ethiopia; ${ }^{2}$ Department of Anesthesia and \\ Critical Care, College of Medicine and \\ Health Science, University of Gondar, \\ Gondar, Ethiopia
}

Background: Female breast cancer is becoming an emerging public health problem which accounts for $33 \%$ of all cancers in women and $23 \%$ of all cancer cases in Ethiopia. The majority of women with breast cancer are diagnosed at later stages due to delayed presentation to seek treatment.

Objective: To determine the prevalence and factors associated with patient delay at presentation among breast cancer patients at Dessie Referral Hospital, the only oncology center in North East Ethiopia.

Methods: We conducted an institution-based cross-sectional study among 204 female patients with pathology-confirmed breast cancer at the only oncology center of North East Ethiopia from January to June 2020. An interviewer administered questionnaire and a medical record data extraction tool were used to address the objective of the study. Patients were said to be delayed for diagnosis if the time duration between first clinical presentations to first clinical consultation was more than 3 months. Then, bivariable and multivariable logistic regression was employed to analyze the association between dependent and independent variables.

Results: Among a total of 209 eligible participants, 5 refused to participate with a response rate of $97.6 \%$. The proportion of patients with delayed presentation was 103 (50.5\%), with the median time taken to visit a healthcare provider after recognition of the first symptom was 4 months. Age above 40 years (AOR $=4.81 ; 95 \% \mathrm{CI}=1.26-18.65) P<0.024$, college and above educational status $(\mathrm{AOR}=0.05 ; 95 \% \mathrm{CI}=0.01-0.77) p<0.036$, government employee $(\mathrm{AOR}=0.19,95 \% \mathrm{CI}=0.03-0.91) \quad P<0.002$, urban residence $(\mathrm{AOR}=0.21 ; 95 \% \mathrm{CI}=0.01-$ 0.82) $p<0.001$, visit traditional healer $(\mathrm{AOR}=0.38 ; 95 \% \mathrm{CI}=0.2-0.69) \quad P<0.0037$, and no lump in under armpit $(\mathrm{AOR}=9.05 ; 95 \% \mathrm{CI}=1.14-22.69) \quad P<0.002$ were associated with delayed presentation.

Conclusion: Delays to seek treatment is generally high in our study. Age, educational status, occupation, residence, visiting traditional healer, and absence of lump in under armpit were significant factors for delayed presentation. Intervention programs focusing on reducing delayed presentation should be employed.

Keywords: breast cancer, delayed presentation, North East Ethiopia

\section{Introduction}

Breast cancer (BC) is the most commonly diagnosed cancer and leading cause of death among women worldwide, with an estimated 2.3 million new cases representing $11.7 \%$ of all cancer cases and more than 685,000 deaths in $2020 .{ }^{1} \mathrm{BC}$ is also the most frequently diagnosed and the leading cause of death in sub-Saharan Africa
Hassen

Department of Public Health, School of

Public Health, College of Medicine and

Health Science, Wollo University, PO Box

342, Dessie, Ethiopia

Tel +25I 92125747I

Email anisa.moh26@gmail.com
Journal of Multidisciplinary Healthcare 2021:14 68I-694

681

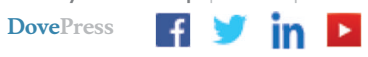

htp:/10i.org/10.214 (c) (7) (5) 2021 Hassen et al. This work is published and licensed by Dove Medical Press Limited. The full terms of this license are available at https://www.dovepress.com/terms. (c) ${ }_{\mathrm{BY}} \mathrm{NC}$ php and incorporate the Creative Commons Attribution - Non Commercial (unported, v3.0) License (http://creativecommons.org/licenses/by-nc/3.0/). By accessing the work you hereby accept the Terms. Non-commercial uses of the work are permitted without any further permission from Dove Medical Press Limited, provided the work is properly attributed. For permission for commercial use of this work, please see paragraphs 4.2 and 5 of our Terms (https://www.dovepress.com/terms.php). 
with an estimated incidence and mortality rate of 22.4 and 17.9 per 100,000 populations respectively in $2020{ }^{1,2}$ According to the Addis Ababa cancer registry, BC constituted $33 \%$ of cancers in women and $23 \%$ of all cancers in Ethiopia. ${ }^{3}$

In high income countries, more than $70 \%$ of $\mathrm{BC}$ patients are diagnosed at an early stage (stages I and II), and prognosis is good; however, in low and middle income countries (LMCs), the infrastructure and resources for routine screening mammography are often unavailable, and $\mathrm{BCs}$ are commonly diagnosed at late stages with only $20-60 \%$ of patients diagnosed in early stages of the disease. ${ }^{4-6}$ Advanced stage diagnosis is associated with decreased survival time and increased mortality rates. ${ }^{5,7}$ Due to this reason, mortality rates are often much higher in LMIC and survival is poorer compared with rates in developed countries..$^{8-10}$

The morbidity and mortality associated with $\mathrm{BC}$ can be mitigated through early detection and receipt of evidencebased, high-quality care. ${ }^{11}$ However, in Ethiopia, a large proportion of $\mathrm{BC}$ patients were diagnosed at an advanced stage, ${ }^{12}$ mainly because of delayed presentation to the health facility after recognition of first symptoms and provider/health system delays in referral of patients to cancer treatment centers average 7 to 18 months. ${ }^{13,14}$

The Aarhus statement defines patient delay presentation as the time interval from the first date of recognition of the first symptom to first clinical presentation or date of the patient first presenting to the health facility. ${ }^{15}$ Different factors have been identified in different studies for the increment of delayed presentation or lack of early healthcare seeking practice after recognition of first symptoms. These factors include: advanced age, ${ }^{16,17}$ low literacy rates, ${ }^{16,18-21}$ lower socioeconomic status, ${ }^{16,22-24}$ being single, ${ }^{19,20}$ residing in a rural area, ${ }^{20,21,25}$ and negative family history ${ }^{16,19}$ among the socio-demographic factors that are responsible for delayed presentation. Lack of breast self-examination, ${ }^{20,25-27}$ lack of knowledge regarding the illness, ${ }^{22,24,27}$ ignorance, ${ }^{19,24,26,28}$ fear of cancer treatment and outcome, ${ }^{22,27-29}$ lack of adequate healthcare service, ${ }^{28,30}$ and use of alternative therapies (traditional medicine and spiritual acts) ${ }^{15,22,24-26,29}$ are also important factors for delayed presentation.

The Ethiopian government drafted the Ethiopian National Cancer Control plan in 2015 for the period of 2016-2020 aimed at improving early diagnosis of breast cancer by promoting breast self-awareness and breast examination for all women coming to the health institution for another purpose. ${ }^{31}$ In addition, the government has expanded the chemotherapy treatment centre in five regions, trained more health professionals, and collaborated with different stakeholders to build the capacity of the healthcare providers. Understanding the recent specific barriers to early diagnosis that Ethiopian breast cancer patients are facing is important for planning targeted interventions that help to mitigate premature death of women from breast cancer.

Therefore, this study aims to investigate the prevalence and factors associated with delayed presentation among breast cancer patients using a quantitative method in Dessie Referral Hospital, the only oncology unit in North East Ethiopia.

\section{Methods}

\section{Study Design and Setting}

An institution-based cross-sectional study design was employed among 204 women with breast cancer at Dessie Referral Hospital (DRH), the only oncology centre in North East Ethiopia, from January to June 2020. DRH is one of the referral hospitals in Amhara region with about 9 million catchment populations who came from more than $200 \mathrm{~km}$ away and is used as a teaching hospital for health science students. The hospital oncology centre started chemotherapy treatment for breast cancer patients since December 2018, and treated approximately 40 breast cancer patients per month according to the center's cancer registry.

\section{Data Collection Procedure and Participants}

All breast cancer patients who were $18^{+}$years and had a clear pathological diagnosis of staging (stage I-IV) (newly diagnosed and on follow up treatment) attending the oncology centre during the data collection period were enrolled consecutively. Those women with breast cancer who were critically ill and unable to communicate during the data collection were excluded from the study.

The data were collected using a structured and pretested questionnaire adapted from reviewing different literatures which can address the objective of the study. Delayed presentation was the dependent variable, while socio-demographic variables like age, level of education, marital status, occupation, residence, number of children, time to come to nearby health facility and referral hospital, and household income; clinical variables like 
hypertension, DM, HIV, and clinical staging; patient history on the diagnostic pathway (dates of first symptom recognition and first healthcare visit, first symptom interpretation, reasons to visit healthcare provider, number of consultations before coming to the hospital, use of traditional medicine, family history of breast cancer, and breast self-examination) and health system factors like type of health service at first consultation, breast examination performed by the physician at first contact, and recommendation of healthcare provider are the independent factors. Household income was collected in Ethiopian birr with currency of 1 US dollar $=37.8$ ETB at the time of data collection. The data were collected through face to face interview and clinical data were extracted from patient's medical charts.

Even though there is no single cut-off point for delayed presentation, this study was based on the recommendation of the Aarhus statement. ${ }^{15}$ Based on this recommendation, waiting for $>3$ months before consulting a healthcare provider was considered as delayed presentation. Patients were retrospectively asked about the breast cancer diagnosis pathway from their first recognition of symptoms to first healthcare visit. We have used local events and holidays to make them remember the dates.

To maintain the quality of data, the data were collected by two BSc nurses from the oncology unit. Training was given to the data collectors and supervisors about the data collection, interviewing, and research ethics. Continuous monitoring and supervision was done by the principal investigator every day for completeness and clarification of the data. Then, the collected data were revised and possible errors were returned to the data collectors for correction on a daily basis. The questionnaire was first developed in English and translated to Amharic, and then translated back to English for consistency (Supplementary Materials). In addition, the questionnaire was pretested on $5 \%$ of breast cancer patients and appropriate corrections were made.

\section{Statistical Analysis}

Data were entered into EpiData 4.2 then exported to STATA 14 software for cleaning and further analysis. Simple descriptive statistics such as frequencies, means, and standard deviations were calculated. Then, to select the variables which are eligible for the multivariable logistic regression model, binary logistic regression was performed and those variables with $p<0.25$ were taken into the final model which is a multivariable logistic regression model. As a result, crude and adjusted odds ratio with $95 \%$ confidence interval was calculated using a backward selection method. A $p$-value of less than or equal to 0.05 was declared significant.

\section{Ethical Consideration}

Ethical clearance and approval was obtained from the institutional review board of Wollo University, College of Health Sciences with protocol number CMHS 5061/ 13/12. The study was conducted in accordance with the declaration of Helsinki and consent was taken from the patients by clearly informing the aim of the study and the information required from them and that being the study subject will have neither financial benefit nor clinical impact. Their right not to be involved in the study was guaranteed without affecting the quality of care delivered to them and the right to withdraw from the interview at any time they want was observed throughout the study. Informed written consent was taken from the study participants and was kept anonymous throughout data collection.

\section{Result}

\section{Socio-Demographic Characteristics}

Out of 209 eligible participants, 5 women with BC refused to participate in the study giving a response rate of $97.6 \%$. Of which 60 (29.4\%) were unable to read and write and 53 $(25.9 \%)$ were married. The mean age was 44.1 years (SD: $11.8)$, and $109(53.4 \%)$ were above 40 . Half of them 98 (48\%) were housewives and $106(52 \%)$ were from an urban residence. Of all 177 (86.8\%) women who gave birth, 85 (48\%) had $\leq 3$ children. Of all patients, 78 (38.0\%) reported average monthly income between 1201 and 2500 birr per month and the median income was 2000 birr. The mean time taken to go to nearby health center was 32.64 (15.02) minutes and it took $<4$ hours to go to referral hospital for nearly half $112(54.9 \%)$ of patients (Table 1 shows the socio-demographic characteristics of women with $\mathrm{BC}$ at $\mathrm{DRH})$.

\section{Clinical Characteristics and Awareness of BC Among Women with Breast Cancer}

More than half of BC patients 135 (66.2\%) have not heard about the disease before their illness. Out of 134 (65.69\%) women with late stage diagnosis, a quarter of them 53 (25.5\%) were diagnosed with stage III and $82(40.2 \%)$ stage IV. Only $12(5.9 \%)$ were diagnosed with stage I at 
Table I Socio-Demographic Characteristics of Women with Breast Cancer at the Only Oncology Center of North East Ethiopia, $2020(N=204)$

\begin{tabular}{|c|c|c|}
\hline Variables & Category & $\mathbf{N}(\%)$ \\
\hline \multirow[t]{5}{*}{ Age } & $20-29$ & $33(16.2)$ \\
\hline & $30-39$ & $62(30.4)$ \\
\hline & $40-49$ & $57(27.9)$ \\
\hline & $50-59$ & $30(14.7)$ \\
\hline & $60-69$ & $22(10.8)$ \\
\hline Age mean $(S D)$ & \multicolumn{2}{|c|}{$44.1(11.8)$} \\
\hline \multirow[t]{4}{*}{ Level of education } & Unable to read and write & $60(29.4)$ \\
\hline & Completion of primary education & $59(28.9)$ \\
\hline & Completion of secondary education & $50(24.5)$ \\
\hline & College and above & $35(17.2)$ \\
\hline \multirow[t]{4}{*}{ Occupational status } & Housewife & $98(48.0)$ \\
\hline & Employed & $46(22.6)$ \\
\hline & Merchant & $32(15.7)$ \\
\hline & Farmer & $28(13.7)$ \\
\hline \multirow[t]{4}{*}{ Marital status } & Single & $19(9.3)$ \\
\hline & Married & $53(25.9)$ \\
\hline & Divorced & $84(4 \mid .2)$ \\
\hline & Widowed & $48(23.6)$ \\
\hline \multirow[t]{2}{*}{ Residence } & Rural & $98(48.0)$ \\
\hline & Urban & $106(52.0)$ \\
\hline \multirow[t]{2}{*}{ Gave birth } & Yes & $177(86.8)$ \\
\hline & No & $27(\mid 3.2)$ \\
\hline \multirow[t]{3}{*}{ Number of children } & $\leq 3$ & $85(48.0)$ \\
\hline & $3-5$ & $56(31.6)$ \\
\hline & $>5$ & $36(20.4)$ \\
\hline \multirow[t]{4}{*}{ Household income } & $\leq 1250$ & $5 I(25.0)$ \\
\hline & $1250-2000$ & $78(38.0)$ \\
\hline & $200 I-3000$ & $27(13.0)$ \\
\hline & $>3001$ & $48(23.0)$ \\
\hline \multirow[t]{3}{*}{ Time to come to DRH (hours) } & $<4$ & $112(54.9)$ \\
\hline & $4-8$ & $64(31.4)$ \\
\hline & $8-12$ & $28(13.7)$ \\
\hline Time to come to nearby health center mean (SD) & \multicolumn{2}{|c|}{$32.64(15.05)$} \\
\hline
\end{tabular}

first presentation. Out of $28(13.7 \%)$ women with breast cancer who had comorbidity, 8 (28.5) were living with HIV and the rest $20(71.5 \%)$ had hypertension. Out of $11(5.4 \%)$ women who had family history of breast cancer, only 3 $(27.2 \%)$ of them performed breast self-examination. Breast lump was the first presenting symptom of their illness for 117 (57.4\%) patients, followed by breast pain and lump in under armpit $70(34.3 \%)$ and $30(14.7 \%)$ respectively (Table 2 shows the clinical characteristics and awareness of $\mathrm{BC}$ among women with breast cancer).

The median time taken to visit a healthcare provider after recognition of the first symptom was 4 months and 103 $(50.5 \%)$ women were delayed for $>3$ months. Out of these delayed women, $75(72.8 \%)$ were diagnosed at late stage $(p<0.030)$. The reasons given by patients for seeking healthcare late were considering the disease as nothing serious 185 
Table 2 Clinical Characteristics and Awareness of Women with Breast Cancer at the Only Oncology Center of North East Ethiopia, $2020(N=204)$

\begin{tabular}{|l|l|c|}
\hline Variables & \multicolumn{1}{|c|}{ Category } & N (\%) \\
\hline Have you heard of breast cancer & Yes & $69(33.8)$ \\
& No & $135(66.2)$ \\
\hline Breast self-examination & Yes & 1 (I) (5.4) \\
& No & $193(94.6)$ \\
\hline Family history of breast cancer & Yes & 1 I (5.4) \\
& No & $193(94.6)$ \\
\hline Comorbid disease & Yes & $28(13.7)$ \\
& No & $176(86.2)$ \\
\hline Stage at diagnosis & Stage I & $12(5.9)$ \\
& Stage II & $57(27.9)$ \\
& Stage III & $53(25.5)$ \\
& Stage IV & $82(40.2)$ \\
\hline First change of breast & Breast lump & $117(57.4)$ \\
& Breast pain & $70(34.3)$ \\
& Swelling & $21(10.3)$ \\
\hline
\end{tabular}

(90.7\%), followed by not having enough awareness about the breast symptoms 113 (55.4\%) and visiting a traditional healer $68(33.3 \%)$. Persistence of the symptom was the main reason to seek a healthcare provider for 200 (98\%) patients (Table 3 ).

The larger proportion $71(68.9 \%)$ of patients aged $\geq 40$ years delayed at presentation as compared with those less than 40 years old $32(31.1 \%)(P<0.001)$. A higher proportion $61(59.2 \%)$ of rural resident women were significantly delayed than their counterparts $(P<0.001)$. A significantly large proportion $42(40.8 \%)$ of illiterate women were also delayed at presentation than those who had primary 35 (33.9\%) and secondary and above educational levels 26 (35.2\%) $(P<0.001)$ (Table 3).

More than half 111 (54.4\%) of the BC patients went to a nearby health center to seek medical treatment for the first time. Out of $70(34.3 \%)$ women who visited $\geq 3$ health facilities, 35 (50\%) were delayed to come to this hospital $(p<0.002)$. Breast examination was performed for almost all (97.1\%) of them at initial consultation and biopsy/FNAC was advised for 139 (68.1\%) (Table 3).

\section{Factors Associated with Delayed Presentation}

Binary and multivariable logistic regression analysis had been performed to assess the association, control confounding variables and identify the strength of association between the dependent variable delayed presentation with independent variables.

In the binary logistic regression (Table 4), patients aged $>40$ years $(\mathrm{COR}=3.67,95 \% \mathrm{CI}=2.05-6.56)$, single marital status $(\mathrm{COR}=3.06,95 \% \mathrm{CI}=1.03-9.11)$, parity of $\geq 3(\mathrm{COR}=1.2,95 \% \mathrm{CI}=1.1-6.15)$, and more than 3 consultations before coming to the hospital ( $\mathrm{COR}=2.05,95 \%$ $\mathrm{CI}=0.98-4.27)$ were significantly associated with delay presentation than their counterparts. Those with college and above level of education $(\mathrm{COR}=0.08,95 \% \mathrm{CI}=0.03-$ $0.25)$, employed women $(\mathrm{COR}=0.19,95 \% \mathrm{CI}=0.09-0.44)$, urban residence $(\mathrm{COR}=0.39,95 \% \mathrm{CI}=0.23-0.69),>3001$ household income $(\mathrm{COR}=0.38,95 \% \mathrm{CI}: 0.17-0.86)$, and not visiting a traditional healer $(\mathrm{COR}=0.38,95 \% \mathrm{CI}=0.21-$ $0.69)$ were also significantly associated with delayed presentation (crude odds ratio (COR) and 95\% confidence interval $(95 \% \mathrm{CI})$ are shown in Table 4$)$.

Variables significantly associated in the binary logistic analysis were further explored in the multivariable logistic regression (Table 5). As a result, only age, educational status, occupation, residence, visiting traditional healer, lump in under armpit, and comorbidity become important factors for delayed presentation. BC patients aged 40 and above years were 4.8 times more 
Table 3 Comparison of Socio-Demographic and Clinical Characteristics, Awareness, Personal and Health System Factors with Delayed Presentation Among BC Patients at the Only Oncology Center of North East Ethiopia, 2020 (N=204)

\begin{tabular}{|c|c|c|c|c|}
\hline \multirow{2}{*}{$\begin{array}{l}\text { Variable } \\
\text { Socio-Demographic Characteristics }\end{array}$} & \multicolumn{2}{|c|}{ Patient Delay } & \multirow[t]{2}{*}{ Chi-Square } & \multirow[t]{2}{*}{$P$ value } \\
\hline & $<3$ Months & $\geq 3$ Months & & \\
\hline \multicolumn{5}{|l|}{ Age } \\
\hline$<40$ & $63(62.4)$ & $32(31.1)$ & 20.08 & $<0.00 I^{*}$ \\
\hline$\geq 40$ & $38(37.6)$ & $71(68.9)$ & & \\
\hline \multicolumn{5}{|l|}{ Level of education } \\
\hline Unable to read and write & $18(17.8)$ & $42(40.8)$ & 28.74 & $<0.001 *$ \\
\hline Completion of primary education & $24(23.8)$ & $35(33.9)$ & & \\
\hline Completion of secondary education & $30(29.7)$ & $20(19.4)$ & & \\
\hline College and above & $29(28.7)$ & $6(5.8)$ & & \\
\hline \multicolumn{5}{|l|}{ Occupation } \\
\hline Housewife & $38(37.6)$ & $60(58.3)$ & 17.94 & $<0.00 I^{*}$ \\
\hline Employed & $35(34.7)$ & II (I0.7) & & \\
\hline Merchant & $14(13.8)$ & $18(17.5)$ & & \\
\hline Farmer & $14(13.8)$ & $14(13.6)$ & & \\
\hline \multicolumn{5}{|l|}{ Residence } \\
\hline Rural & $37(36.6)$ & $61(59.2)$ & 10.42 & $0.001 *$ \\
\hline Urban & $64(63.4)$ & $42(40.8)$ & & \\
\hline \multicolumn{5}{|l|}{ Marital status } \\
\hline Single & $12(11.8)$ & $7(6.8)$ & 6.30 & 0.098 \\
\hline Married & $19(18.8)$ & $34(33.1)$ & & \\
\hline Divorced & $46(45.5)$ & $38(36.9)$ & & \\
\hline Widowed & $24(23.8)$ & $24(23.3)$ & & \\
\hline \multicolumn{5}{|l|}{ Parity } \\
\hline$\leq 3$ & $49(59.0)$ & $36(38.3)$ & 7.91 & 0.789 \\
\hline $3-5$ & $22(26.5)$ & $34(36.2)$ & & \\
\hline$>5$ & $12(14.5)$ & $24(25.5)$ & & \\
\hline \multicolumn{5}{|l|}{ Household income } \\
\hline$\leq 1250$ & $21(20.8)$ & $30(29.1)$ & 10.56 & 0.103 \\
\hline $1250-2000$ & $37(36.6)$ & $41(39.8)$ & & \\
\hline $200 I-3000$ & $12(11.8)$ & $15(14.6)$ & & \\
\hline$>3001$ & $31(30.7)$ & $17(16.5)$ & & \\
\hline \multicolumn{5}{|l|}{ Time to come to DRH } \\
\hline$<4$ & $57(56.4)$ & $55(53.4)$ & 5.78 & 0.875 \\
\hline $4-8$ & $30(29.7)$ & $34(33.1)$ & & \\
\hline $8-12$ & $14(13.8)$ & $14(13.6)$ & & \\
\hline \multicolumn{5}{|l|}{ Awareness of breast cancer } \\
\hline Yes & $21(20.8)$ & $9(8.7)$ & 10.29 & $0.00 I^{*}$ \\
\hline No & $80(79.2)$ & $94(91.3)$ & & \\
\hline
\end{tabular}

(Continued) 
Table 3 (Continued).

\begin{tabular}{|c|c|c|c|c|}
\hline \multirow{2}{*}{$\begin{array}{l}\text { Variable } \\
\text { Socio-Demographic Characteristics }\end{array}$} & \multicolumn{2}{|c|}{ Patient Delay } & \multirow[t]{2}{*}{ Chi-Square } & \multirow[t]{2}{*}{$P$ value } \\
\hline & $<3$ Months & $\geq 3$ Months & & \\
\hline \multicolumn{5}{|l|}{ Clinical characteristics } \\
\hline \multicolumn{5}{|l|}{ Comorbidity } \\
\hline Yes & $10(9.9)$ & $18(17.5)$ & 2.47 & 0.116 \\
\hline No & $91(90.1)$ & $85(82.5)$ & & \\
\hline \multicolumn{5}{|l|}{ Stage at diagnosis } \\
\hline Early stage & $42(41.6)$ & $28(27.2)$ & 4.69 & $0.030 *$ \\
\hline Late stage & $59(58.4)$ & $75(72.8)$ & & \\
\hline \multicolumn{5}{|l|}{ First change of breast } \\
\hline \multicolumn{5}{|l|}{ Breast lump } \\
\hline Yes & $56(55.5)$ & 61 (59.2) & 0.29 & 0.586 \\
\hline No & $45(44.5)$ & $42(40.8)$ & & \\
\hline \multicolumn{5}{|l|}{ Breast pain } \\
\hline Yes & $44(43.6)$ & $26(25.2)$ & 7.59 & $0.006^{*}$ \\
\hline No & $57(56.4)$ & $77(74.8)$ & & \\
\hline \multicolumn{5}{|l|}{ Lump in under armpit } \\
\hline Yes & $21(20.8)$ & $9(8.74)$ & 5.91 & $0.015^{*}$ \\
\hline No & $80(79.2)$ & $94(91.3)$ & & \\
\hline \multicolumn{5}{|l|}{ Personal factors for delay } \\
\hline Interpret symptom as nothing serious & $85(84.2)$ & $100(97.1)$ & 10.5 & $<0.001$ \\
\hline Lack of awareness about the symptom & $50(49.5)$ & $63(6 I .2)$ & & \\
\hline Visit traditional healer & $23(22.7)$ & $45(43.7)$ & & \\
\hline Lack of money & $24(23.7)$ & $41(39.8)$ & & \\
\hline Thought it will relief by itself & $91(90.1)$ & $95(92.2)$ & & \\
\hline \multicolumn{5}{|l|}{ Family history } \\
\hline Yes & $4(3.96)$ & $7(6.8)$ & 0.81 & 0.370 \\
\hline No & $97(96.1)$ & $96(93.2)$ & & \\
\hline \multicolumn{5}{|l|}{ Health system factors for delay } \\
\hline \multicolumn{5}{|l|}{ Recommendation of healthcare provider } \\
\hline Nothing series & $23(22.7)$ & $22(21.3)$ & 1.03 & 0.597 \\
\hline Infection & $14(13.8)$ & $10(9.7)$ & & \\
\hline Maybe cancer & $64(63.7)$ & $71(68.9)$ & & \\
\hline \multicolumn{5}{|l|}{ Referred by the clinician } \\
\hline Yes & $64(63.4)$ & $71(68.9)$ & 0.77 & 0.401 \\
\hline No & $37(36.6)$ & $32(31.1)$ & & \\
\hline \multicolumn{5}{|c|}{ Number of consultations before coming to DRH } \\
\hline $0-1$ & $37(36.6)$ & $18(17.5)$ & 12.12 & $0.002^{*}$ \\
\hline 2 & $29(28.7)$ & $50(48.5)$ & & \\
\hline$\geq 3$ & $35(34.6)$ & $35(33.9)$ & & \\
\hline
\end{tabular}

Note: *Statistically significant at $p<0.05$. 
Table 4 Binary Logistic Regression Analysis of Variables Associated with Delayed Presentation Among BC Patients at the Only Oncology Center of North East Ethiopia, $2020(\mathrm{~N}=204)$

\begin{tabular}{|c|c|c|c|c|}
\hline \multirow{2}{*}{$\begin{array}{l}\text { Variable } \\
\text { Socio-Demographic Characteristics }\end{array}$} & \multicolumn{2}{|c|}{ Patient Delay } & \multirow[t]{2}{*}{ COR $(95 \% \mathrm{Cl})$} & \multirow[t]{2}{*}{$P$ value } \\
\hline & $<3$ Months & $\geq 3$ Months & & \\
\hline \multicolumn{5}{|l|}{ Age } \\
\hline$<40$ & $63(62.4)$ & $32(31.1)$ & 1 & \\
\hline$\geq 40$ & $38(37.6)$ & 71 (68.9) & $3.67^{*}(2.05-6.56)$ & $0.021 *$ \\
\hline \multicolumn{5}{|l|}{ Level of education } \\
\hline Unable to read and write & $18(17.8)$ & $42(40.8)$ & 1 & \\
\hline Completion of primary education & $24(23.8)$ & $35(33.9)$ & $0.62(0.29-1.33)$ & 0.118 \\
\hline Completion of secondary education & $30(29.7)$ & $20(19.4)$ & $0.28 *(0.12-0.62)$ & $0.036^{*}$ \\
\hline College and above & $29(28.7)$ & $6(5.8)$ & $0.08 *(0.03-0.25)$ & $0.031 *$ \\
\hline \multicolumn{5}{|l|}{ Occupation } \\
\hline Housewife & $38(37.6)$ & $60(58.3)$ & 1 & \\
\hline Employed & $35(34.7)$ & II (I0.7) & $0.19 *(0.09-0.44)$ & $0.002^{*}$ \\
\hline Merchant & $14(13.8)$ & $18(17.5)$ & $0.81(0.36-1.82)$ & 0.757 \\
\hline Farmer & $14(13.8)$ & $14(13.6)$ & $0.63(0.27-1.47)$ & 0.298 \\
\hline \multicolumn{5}{|l|}{ Residence } \\
\hline Rural & $37(36.6)$ & $61(59.2)$ & $\mathrm{I}$ & \\
\hline Urban & $64(63.4)$ & $42(40.8)$ & $0.39 *(0.23-0.69)$ & $0.00 I^{*}$ \\
\hline \multicolumn{5}{|l|}{ Marital status } \\
\hline Single & $12(11.8)$ & $7(6.8)$ & 1 & \\
\hline Married & $19(18.8)$ & $34(33.1)$ & $3.06(1.03-9.11)$ & $0.043^{*}$ \\
\hline Divorced & $46(45.5)$ & $38(36.9)$ & $1.4 \mid(0.5 I-3.95)$ & 0.506 \\
\hline Widowed & $24(23.8)$ & $24(23.3)$ & I.7I $(0.57-5.10)$ & 0.333 \\
\hline \multicolumn{5}{|l|}{ Parity } \\
\hline$\leq 3$ & $49(59.0)$ & $36(38.3)$ & 1 & \\
\hline $3-5$ & $22(26.5)$ & $34(36.2)$ & $2.10(1.05-4.18)$ & $0.034^{*}$ \\
\hline$>5$ & $12(14.5)$ & $24(25.5)$ & $1.2(1.1-6.15)$ & $0.016^{*}$ \\
\hline \multicolumn{5}{|l|}{ Household income } \\
\hline$\leq 1250$ & $21(20.8)$ & $30(29.1)$ & 1 & \\
\hline $1250-2000$ & $37(36.6)$ & $41(39.8)$ & $0.78(0.38-1.58)$ & 0.485 \\
\hline $200 I-3000$ & $12(11.8)$ & $15(14.6)$ & $0.87(0.34-2.24)$ & 0.781 \\
\hline$>3001$ & 31 (30.7) & $17(16.5)$ & $0.38(0.17-0.86)$ & $0.021 *$ \\
\hline \multicolumn{5}{|l|}{ Time to come to DRH } \\
\hline$<4$ & $57(56.4)$ & $55(53.4)$ & I & \\
\hline $4-8$ & $30(29.7)$ & $34(33.1)$ & $1.17(0.63-2.17)$ & 0.608 \\
\hline $8-12$ & $14(13.8)$ & $14(13.6)$ & $1.03(0.45-2.37)$ & 0.933 \\
\hline \multicolumn{5}{|l|}{ Awareness of breast cancer } \\
\hline Yes & $21(20.8)$ & $9(8.7)$ & 1 & \\
\hline No & $80(79.2)$ & $94(91.3)$ & $2.74(1.18-6.32)$ & $0.037^{*}$ \\
\hline
\end{tabular}


Table 4 (Continued).

\begin{tabular}{|c|c|c|c|c|}
\hline \multirow{2}{*}{$\begin{array}{l}\text { Variable } \\
\text { Socio-Demographic Characteristics }\end{array}$} & \multicolumn{2}{|c|}{ Patient Delay } & \multirow[t]{2}{*}{ COR $(95 \% \mathrm{Cl})$} & \multirow[t]{2}{*}{$P$ value } \\
\hline & $<3$ Months & $\geq 3$ Months & & \\
\hline \multicolumn{5}{|l|}{ Comorbidity } \\
\hline Yes & $10(9.9)$ & $18(17.5)$ & 1 & \\
\hline No & $91(90.1)$ & $85(82.5)$ & $0.52(0.23-1.19)$ & $0.120 *$ \\
\hline \multicolumn{5}{|l|}{ Stage at diagnosis } \\
\hline Early stage & $42(41.6)$ & $28(27.2)$ & 1 & \\
\hline Late stage & $59(58.4)$ & $75(72.8)$ & $1.91(1.05-3.43)$ & 0.031 \\
\hline \multicolumn{5}{|l|}{ First change of breast } \\
\hline \multicolumn{5}{|l|}{ Breast lump } \\
\hline Yes & $56(55.5)$ & 61 (59.2) & 1 & \\
\hline No & $45(44.5)$ & $42(40.8)$ & $0.85(0.49-1.49)$ & 0.586 \\
\hline \multicolumn{5}{|l|}{ Breast pain } \\
\hline Yes & $44(43.6)$ & $26(25.2)$ & 1 & \\
\hline No & $57(56.4)$ & $77(74.8)$ & $2.28(1.26-4.13)$ & $0.006^{*}$ \\
\hline \multicolumn{5}{|l|}{ Lump in under armpit } \\
\hline Yes & $21(20.8)$ & $9(8.74)$ & I & \\
\hline No & $80(79.2)$ & $94(91.3)$ & $2.74(1.18-6.32)$ & $0.018^{*}$ \\
\hline \multicolumn{5}{|l|}{ Discharge/ulceration } \\
\hline Yes & $9(8.9)$ & $6(5.8)$ & I & \\
\hline No & $92(91.0)$ & 97 (94.2) & $1.58(0.54-4.61)$ & 0.402 \\
\hline \multicolumn{5}{|l|}{ Pulling in of nipple } \\
\hline Yes & $10(9.9)$ & $7(6.8)$ & 1 & \\
\hline No & $91(90.1)$ & $96(93.2)$ & $1.55(0.55-4.12)$ & 0.4252 \\
\hline \multicolumn{5}{|l|}{ Personal factors for delay } \\
\hline \multicolumn{5}{|l|}{ Lack of money } \\
\hline Yes & $24(23.8)$ & $4 \mid(39.8)$ & $\mathrm{I}$ & \\
\hline No & $77(76.2)$ & $62(60.2)$ & $0.47(0.25-0.86)$ & 0.015 \\
\hline \multicolumn{5}{|l|}{ Lack of awareness about the symptom } \\
\hline Yes & $50(49.5)$ & $63(6 \mid .2)$ & 1 & \\
\hline No & $51(50.5)$ & $40(38.8)$ & $0.62(0.35-1.08)$ & 0.595 \\
\hline \multicolumn{5}{|l|}{ Thought it will relief by itself } \\
\hline Yes & 91 (90.1) & 95 (92.2) & I & \\
\hline No & $10(9.9)$ & $8(7.7)$ & $0.76(0.28-2.02)$ & 0.592 \\
\hline \multicolumn{5}{|l|}{ Sought traditional/spiritual healer } \\
\hline Yes & $23(22.7)$ & 45 (43.7) & I & \\
\hline No & 78 (77.2) & $58(56.3)$ & $0.38(0.21-0.69)$ & 0.002 \\
\hline
\end{tabular}


Table 4 (Continued).

\begin{tabular}{|c|c|c|c|c|}
\hline \multirow{2}{*}{$\begin{array}{l}\text { Variable } \\
\text { Socio-Demographic Characteristics }\end{array}$} & \multicolumn{2}{|c|}{ Patient Delay } & \multirow[t]{2}{*}{ COR $(95 \% \mathrm{Cl})$} & \multirow[t]{2}{*}{$P$ value } \\
\hline & $<3$ Months & $\geq 3$ Months & & \\
\hline \multicolumn{5}{|l|}{ Type of health service at first visit } \\
\hline Health centre & $47(46.5)$ & $64(62.1)$ & I & \\
\hline Private clinic & $27(26.7)$ & $13(12.6)$ & $0.35(0.16-0.75)$ & $0.007^{*}$ \\
\hline Hospital & $27(26.7)$ & $26(25.2)$ & $0.71(0.36-1.36)$ & 0.301 \\
\hline \multicolumn{5}{|c|}{ Recommendation of healthcare provider } \\
\hline Nothing serious & $23(22.8)$ & $22(21.4)$ & I & \\
\hline Infection & $14(13.8)$ & $10(9.71)$ & $0.74(0.27-2.03)$ & 0.567 \\
\hline May be cancer & $64(63.4)$ & 71 (68.9) & I.I5 (0.59-2.27) & 0.667 \\
\hline \multicolumn{5}{|l|}{ Referred by the clinician } \\
\hline Yes & $64(63.4)$ & 71 (68.9) & I & \\
\hline No & $37(36.6)$ & $32(31.1)$ & $0.77(0.43-1.39)$ & 0.401 \\
\hline \multicolumn{5}{|c|}{ Number of consultations before coming to DRH } \\
\hline $0-1$ & $37(36.6)$ & $18(17.5)$ & I & \\
\hline 2 & $29(28.7)$ & $50(48.5)$ & $3.54(1.7 \mid-7.32)$ & $0.00 I^{*}$ \\
\hline$\geq 3$ & $35(34.6)$ & 35 (33.9) & $2.05(0.98-4.27)$ & $0.054^{*}$ \\
\hline
\end{tabular}

Note: *Statistically significant at $p<0.25$.

likely to delay to health center than age less than 40 years $(\mathrm{AOR}=4.81 ; 95 \% \mathrm{CI}=1.26-18.65)$. Besides, $\mathrm{BC}$ patients who had college and above educational status were $95 \%$ less likely to delay at presentation than women who were unable to read and write ( $\mathrm{AOR}=0.05 ; 95 \% \mathrm{CI}=0.01-0.77$ ). Being employed was also an important predictor of delayed presentation in which employed BC patients were 0.14 times less likely to delay housewives $(\mathrm{AOR}=0.19,95 \% \mathrm{CI}=0.03-0.91)$. Similarly, patients who were from an urban residence were $79 \%$ less likely to delay than rural residents (AOR $=0.21 ; 95 \% \mathrm{CI}=0.01-0.82$ ).

Breast cancer patients who have not visited a traditional/spiritual healer were less likely to delay than their counterparts. Those women who did not visit a traditional healer were $62 \%$ less likely to delay than who visited a traditional healer $\quad(\mathrm{AOR}=0.38 ; \quad 95 \% \quad \mathrm{CI}=0.2-0.69)$. Similarly, breast cancer patients who had no lump in under armpit were approximately 9 times more likely to delay than their counterparts $(\mathrm{AOR}=9.05 ; 95 \% \mathrm{CI}=1.14$ 22.69) (Table 5 shows adjusted odds ratio with $95 \%$ confidence interval of multivariable logistic regression analysis).

\section{Discussion}

Our study assessed delayed presentation of women with breast cancer and its associated factors at Dessie Referral Hospital, the only oncology unit in North East Ethiopia. The study revealed that the median time taken to consult a health professional after noticing the first symptom was 4 months. The finding is in line with a multi-national analysis survey ${ }^{32}$ and other studies conducted in low and middle income countries like Mali, Rwanda, and Senegal. ${ }^{21,25,33}$ However, it was shorter than studies in Nigeria and Libya $^{26,34}$ which is 17 months and 7.5 months respectively. In contrast, it is longer than studies conducted in Brazil, China, and Egypt. ${ }^{18,35,36}$ The discrepancy might be due to the difference in knowledge and awareness status of the study population among countries. As Ethiopia is a developing country with low awareness about noncommunicable disease including cancer, women may present late to a health facility. ${ }^{31}$ Besides, in this study, a large proportion of women with breast cancer have not had enough awareness about the symptoms before their diagnosis, resulting in delayed patient presentation to seek treatment.

The proportion of patient delay accounts for 103 $(50.3 \%)$, which is higher than studies in Pakistan, Iran, 
Table 5 Factors Associated with Delayed Presentation of Women with Breast Cancer at the Only Oncology Center of North East Ethiopia, $2020(\mathrm{~N}=204)$

\begin{tabular}{|c|c|c|c|c|c|}
\hline \multirow[t]{2}{*}{ Variable } & \multicolumn{2}{|c|}{ Patient Delay } & \multirow[t]{2}{*}{ COR $(95 \% \mathrm{Cl})$} & \multirow[t]{2}{*}{ AOR $(95 \% \mathrm{Cl})$} & \multirow[t]{2}{*}{$P$ value } \\
\hline & $<3$ Months & $\geq 3$ Months & & & \\
\hline \multicolumn{6}{|l|}{ Age } \\
\hline$<40$ & $63(62.4)$ & $32(3 I .1)$ & I & 1 & \\
\hline$\geq 40$ & $38(37.6)$ & 71 (68.9) & $3.67(2.05-6.56)$ & $4.81(1.26-18.65)$ & $0.02 I^{*}$ \\
\hline \multicolumn{6}{|l|}{ Level of education } \\
\hline Unable to read and write & $18(17.8)$ & $42(40.8)$ & I & & \\
\hline Secondary not completed & $24(23.8)$ & $35(33.9)$ & $0.62(0.29-1.33)$ & $0.42(0.14-1.24)$ & 0.118 \\
\hline Secondary education completed & $30(29.7)$ & $20(19.4)$ & $0.28(0.12-0.62)$ & $0.2(0.05-0.94)$ & $0.036^{*}$ \\
\hline College and above & $29(28.7)$ & $6(5.8)$ & $0.08(0.03-0.25)$ & $0.05(0.01-0.77)$ & $0.03 I^{*}$ \\
\hline \multicolumn{6}{|l|}{ Occupation } \\
\hline Housewife & $38(37.6)$ & $60(58.3)$ & 1 & & \\
\hline Employed & $35(34.7)$ & II (I0.7) & $0.19(0.09-0.44)$ & $0.14(0.03-0.91)$ & $0.002^{*}$ \\
\hline Private & $14(13.8)$ & $18(17.5)$ & $0.81(0.36-1.82)$ & $0.77(0.14-4.03)$ & 0.757 \\
\hline Farmer & $14(13.8)$ & $14(13.6)$ & $0.63(0.27-1.47)$ & $0.4 \mathrm{I}(0.07-2.2)$ & 0.298 \\
\hline \multicolumn{6}{|l|}{ Residence } \\
\hline Rural & $37(36.6)$ & $61(59.2)$ & 1 & & \\
\hline Urban & $64(63.4)$ & $42(40.8)$ & $0.39(0.23-0.69)$ & $0.21(0.01-0.82)$ & $0.001 *$ \\
\hline \multicolumn{6}{|l|}{ Lump in armpit } \\
\hline Yes & $21(20.8)$ & 9 (8.7) & 1 & & \\
\hline No & $80(79.2)$ & $94(91.3)$ & $2.74(1.18-6.32)$ & 9.05 (1.14-22.69) & $0.037^{*}$ \\
\hline \multicolumn{6}{|l|}{ Visit traditional healer } \\
\hline Yes & $32(31.7)$ & $45(43.7)$ & I & & \\
\hline No & $69(68.3)$ & $58(56.3)$ & $0.38 *(0.2-0.69)$ & $0.05(0.0 \mathrm{I}-0.27)$ & $0.002^{*}$ \\
\hline
\end{tabular}

Note: *Statistically significant at $p<0.05$.

and South Africa, ${ }^{16,37,38}$ however, lower than Senegal, Tanzania, and Nigeria. ${ }^{18,33,39}$ The difference might be due to the difference in sample size of the studies and different use of the cut-off point for classifying patient delay. Besides, large proportions (65.69\%) of women with breast cancer were presented at an advanced stage. This is higher than studies reported in $\operatorname{Egypt}^{36}(60 \%)$ and Morocco $(46 \%) .{ }^{40}$ However, these studies had been conducted with small sample size resulting in shorter median time and small proportion of advanced stage diagnosis.

More than three-quarters of patients with delayed presentation were diagnosed with advanced stages of BC at presentation. It has been reported in studies conducted in South Africa and Ethiopia ${ }^{14,41}$ that delayed presentation will create a good environment for the progression of the disease resulting in advanced stage diagnosis. ${ }^{38,40}$
The main reasons given by women with breast cancer for coming late to the health facility were considering the disease as nothing serious, not having enough awareness about the symptoms, and visiting a traditional healer. These reasons were also mentioned in other areas of the Ethiopia northwest region, Iran, Mali, Pakistan, Nigeria, and Libya. ${ }^{14,16,19,25,26,38}$

Our study showed that age greater than 40, college and above educational status, occupation, urban residence, no lump under armpit, and visiting a traditional healer were significantly associated with patient delay in the multivariable logistic regression.

Age of 40 and above was associated with delayed presentation by 4.8 times. A similar study from $\mathrm{China}^{35}$ also found that aged women tend to present later than their counterparts. Those women of age 40 and above may think the disease symptoms could be results of the aging process 
and menopausal features neglecting the symptom and delay at presentation.

Women who had college and above educational status and employed were $95 \%$ and $86 \%$ less likely to delay than women who were unable to read and write and housewives respectively. Similar findings have also been reported in Pakistan ${ }^{16}$ and Morocco. ${ }^{40}$ This is explained by illiterate and unemployed women not having enough awareness and knowledge about the disease symptoms and severity, as they could not have access to health information, health education, and sociocultural impacts on health-seeking behaviors. Moreover, literacy and unemployment may also affect presentation due to economic and transportation issues. Besides, it has been reported that breast cancer knowledge about early detection and treatment is low in sub-Saharan Africa. ${ }^{27}$

Patients who were from an urban residence were also $79 \%$ less likely to delay than rural residents. Women who come from rural countries may have difficulty of transportation to a nearby health center and referral hospitals, traveling a long distance to get appropriate diagnosis, which in turn may result in delayed presentation. The finding is consistent with previous studies in South Africa, Morocco, and North West part of Ethiopia. ${ }^{14,37,40}$

Breast cancer patients who have not visited a traditional/ spiritual healer before seeking medical treatment were less likely to delay than their counterparts. Those women who did not visit a traditional healer were 0.38 times less likely to delay than who visited a traditional healer. Similarly, findings from Libya $^{26}$ and Rwanda ${ }^{21}$ also show that the main reason given by the patients for their delays was having tried traditional medicine first. Most women in developing countries believe to treat themselves by traditional remedies before going to a health facility. So, while taking those remedies, most patients delay to come to the health facility leading to worsening of symptoms and advanced stage.

Breast cancer patients who had no lump in under armpit were approximately 9 times more likely to delay than their counterparts. Similar findings have been reported in Libya and Ethiopia. ${ }^{14,26}$ This might be because women may perceive their symptom as benign and selflimited and not consider the symptom as severe and affect their daily lives. It may also be due to poor practice of breast self-examination among women resulting in failure to detect breast lump unless advanced resulting in armpit swelling or lump. This may contribute to delayed presentation in seeking medical care.

\section{Strength and Limitations}

This is the first study conducted in the only oncology center of North East Ethiopia to determine the factors associated with presentation delay of breast cancer patients. As a result, its representativeness is high to the region. However, due to the cross-sectional nature of the study and small sample size, the study could not show a cause and effect relationship. Besides, recall bias could be a potential threat to the study, not remembering the exact date of their first symptom recognition, therefore the study should be used cautiously.

\section{Conclusion}

Delayed presentation with advanced breast cancer disease is common in the region. Many factors may contribute to this situation. Age above 40 years, illiteracy, unemployed occupation, rural residence, no lump under armpit, and visiting a traditional healer were the main factors contributing to the delay at presentation to a health facility after recognition of the first symptom.

\section{Abbreviations}

DRH, Dessie Referral Hospital; ETB, Ethiopian birr; LMIC, Lower and middle income countries; NRR, Nonresponse rate; OR, Odds ratio; SPSS, Statistical Package for Social Science.

\section{Data Sharing Statement}

The data for this study will be obtained from the corresponding author upon reasonable request.

\section{Acknowledgment}

We would like to express our deepest gratitude to Dessie Referral Hospital oncology centre staff, data collectors and patients for their cooperation during the data collection period.

\section{Disclosure}

The authors declare no conflicts of interest in this work.

\section{References}

1. Sung H, Ferlay J, Siegel RL, et al. Global cancer statistics 2020: GLOBOCAN estimates of incidence and mortality worldwide for 36 cancers in 185 countries. CA Cancer J Clin. 2021. doi:10.3322/ caac. 21660

2. Adeloye D, Sowunmi OY, Jacobs W, et al. Estimating the incidence of breast cancer in Africa: a systematic review and meta-analysis. $J$ Glob Health. 2018;8(1). doi:10.7189/jogh.08.010419.

3. Timotewos G, Solomon A, Mathewos A, et al. First data from a population based cancer registry in Ethiopia. Cancer Epidemiol. 2018;53:93-98. doi:10.1016/j.canep.2018.01.008 
4. Unger-Saldaña K. Challenges to the early diagnosis and treatment of breast cancer in developing countries. World J Clin Oncol. 2014;5 (3):465. doi:10.5306/wjco.v5.i3.465

5. Brinton LA, Figueroa JD, Awuah B, et al. Breast cancer in SubSaharan Africa: opportunities for prevention. Breast Cancer Res Treat. 2014;144(3):467-478. doi:10.1007/s10549-014-2868-Z

6. Jedy-Agba E, McCormack V, Adebamowo C, dos-Santos-Silva I. Stage at diagnosis of breast cancer in sub-Saharan Africa: a systematic review and meta-analysis. Lancet Glob Health. 2016;4(12):e923e935. doi:10.1016/S2214-109X(16)30259-5

7. Howlader N, Noone A, Krapcho M, et al. SEER cancer statistics review, 1975-2016. National Cancer Institute; 2019.

8. Mattos JSDC, Mauad EC, Syrjänen K, et al. The impact of breast cancer screening among younger women in the Barretos Region, Brazil. Anticancer Res. 2013;33(6):2651-2655.

9. Legesse B, Gedif T. Knowledge on breast cancer and its prevention among women household heads in Northern Ethiopia. Open J Prev Med. 2014;2014.

10. Torre LA, Islami F, Siegel RL, Ward EM, Jemal A. Global cancer in women: burden and trends. Cancer Epidemiol Biomarkers Prev. 2017;26(4):444-457. doi:10.1158/1055-9965.EPI-16-0858

11. World Health Organization. Global Status Report on Noncommunicable Diseases 2014. World Health Organization; 2014.

12. Kantelhardt E, Zerche $P$, Mathewos A, et al. Breast cancer survival in Ethiopia: a cohort study of 1070 women. Int J Cancer. 2014;135 (3):702-709. doi:10.1002/ijc.28691

13. Dye TD, Bogale S, Hobden C, Tilahun Y, Deressa T, Reeler A. Experience of initial symptoms of breast cancer and triggers for action in Ethiopia. Int J Breast Cancer. 2012;2012:1-5. doi:10.1155/2012/908547

14. Tesfaw A, Demis S, Munye T, Ashuro Z. Patient delay and contributing factors among breast cancer patients at two cancer referral centres in Ethiopia: a Cross-Sectional Study. $J$ Multidiscip Healthc. 2020;13:1391. doi:10.2147/JMDH.S275157

15. Weller D, Vedsted P, Rubin G, et al. The Aarhus statement: improving design and reporting of studies on early cancer diagnosis. $\mathrm{Br}$ Cancer. 2012;106(7):1262-1267. doi:10.1038/bjc.2012.68

16. Khan MA, Hanif S, Iqbal S, Shahzad MF, Shafique S, Khan MT. Presentation delay in breast cancer patients and its association with sociodemographic factors in North Pakistan. Chin J Cancer Res. 2015;27(3):288. doi:10.3978/j.issn.1000-9604.2015.04.11

17. Maghous A, Rais F, Ahid S, et al. Factors influencing diagnosis delay of advanced breast cancer in Moroccan women. BMC Cancer. 2016;16(1):1-8. doi:10.1186/s12885-016-2394-y

18. Lopes TCR, Gravena AAF, de Oliveira Demitto M, et al. Delay in diagnosis and treatment of breast cancer among women attending a reference service in Brazil. Asian Pac J Cancer Prev. 2017;18(11):3017.

19. Ibrahim N, Oludara M. Socio-demographic factors and reasons associated with delay in breast cancer presentation: a study in Nigerian women. Breast. 2012;21(3):416-418. doi:10.1016/j.breast.2012.02.006

20. Dianatinasab M, Fararouei M, Mohammadianpanah M, ZareBandamiri M. Impact of social and clinical factors on diagnostic delay of breast cancer: a Cross-sectional Study. Medicine. 2016;95 (38):e4704. doi:10.1097/MD.0000000000004704

21. Pace LE, Mpunga T, Hategekimana V, et al. Delays in breast cancer presentation and diagnosis at two rural cancer referral centers in Rwanda. Oncologist. 2015;20(7):780. doi:10.1634/theoncologist.2014-0493

22. Asoogo C, Duma SE. Factors contributing to late breast cancer presentation for health care amongst women in Kumasi, Ghana. Curationis. 2015;38(1):1-7. doi:10.4102/curationis.v38i1.1287

23. Sathwara JA, Balasubramaniam G, Bobdey SC, Jain A, Saoba S. Sociodemographic factors and late-stage diagnosis of breast cancer in India: a hospital-based study. Indian J Med Paediatr Oncol. 2017;38 (3):277. doi:10.4103/ijmpo.ijmpo_15_16
24. Gebremariam A, Addissie A, Worku A, Assefa M, Kantelhardt EJ, Jemal A. Perspectives of patients, family members, and health care providers on late diagnosis of breast cancer in Ethiopia: a qualitative study. PLoS One. 2019;14(8):e0220769. doi:10.1371/journal.pone.0220769

25. Grosse Frie K, Kamaté B, Traoré CB, et al. Factors associated with time to first healthcare visit, diagnosis and treatment, and their impact on survival among breast cancer patients in Mali. PLoS One. 2018;13 (11):e0207928. doi:10.1371/journal.pone.0207928

26. Ermiah E, Abdalla F, Buhmeida A, Larbesh E, Pyrhönen S, Collan Y. Diagnosis delay in Libyan female breast cancer. BMC Res Notes. 2012;5(1):1-8. doi:10.1186/1756-0500-5-452

27. Akuoko CP, Armah E, Sarpong T, Quansah DY, Amankwaa I, Boateng D. Barriers to early presentation and diagnosis of breast cancer among African women living in sub-Saharan Africa. PLoS One. 2017;12(2):e0171024. doi:10.1371/journal.pone.0171024

28. Dai D. Black residential segregation, disparities in spatial access to health care facilities, and late-stage breast cancer diagnosis in metropolitan Detroit. Health Place. 2010;16(5):1038-1052. doi:10.1016/j. healthplace.2010.06.012

29. Norsa'adah B, Rampal KG, Rahmah MA, Naing NN, Biswal BM. Diagnosis delay of breast cancer and its associated factors in Malaysian women. BMC Cancer. 2011;11(1):1-8. doi:10.1186/ 1471-2407-11-1

30. Pritchard C, Wallace MS. Patient and provider delays in breast cancer patients attending a tertiary care centre: a prospective study. JRSM Short Rep. 2011;2(10):1-4. doi:10.1258/shorts.2010.010079

31. Federal Ministry of Health Ethiopia Disease Prevention and Control Directorate. National cancer control plan 2016-2020; 2015.

32. Jassem J, Ozmen V, Bacanu F, et al. Delays in diagnosis and treatment of breast cancer: a multinational analysis. Eur J Public Health. 2014;24(5):761-767. doi:10.1093/eurpub/ckt131

33. Gueye M, Gueye SMK, Diallo M, et al. Sociodemographic factors associated with delays in breast cancer. Open J Obstet Gynecol. 2017;7(04):455. doi:10.4236/ojog.2017.74047

34. Umoke IC, Garba ES. Breast cancer in North-Central Nigeria: challenges to good management outcome. Int Surg J. 2019;6(9):31053110. doi:10.18203/2349-2902.isj20194042

35. Zhang H, Wang G, Zhang J, Lu Y, Jiang X. Patient delay and associated factors among Chinese women with breast cancer: a cross-sectional study. Medicine. 2019;98(40).

36. Mousa SM, Seifeldin IA, Hablas A, Elbana ES, Soliman AS. Patterns of seeking medical care among Egyptian breast cancer patients: relationship to late-stage presentation. Breast. 2011;20(6):555-561. doi:10.1016/j.breast.2011.07.001

37. Rayne S, Schnippel K, Grover S, Kruger D, Benn C, Firnhaber C. The effect of beliefs about breast cancer on stage and delay to presentation: results from a prospective study in urban South Africa. S Afr J Surg. 2019;57(1):12-18. doi:10.17159/2078-5151/ 2018/v56n2a2704

38. Harirchi I, Karbakhsh M, Hadi F, Madani SS, Sirati F, Kolahdoozan S. Patient delay, diagnosis delay and treatment delay for breast cancer: comparison of the pattern between patients in public and private health sectors. Arch Breast Cancer. 2015:52-57.

39. Mabula JB, Mchembe MD, Chalya PL, et al. Stage at diagnosis, clinicopathological and treatment patterns of breast cancer at Bugando Medical Centre in north-western Tanzania. Tanzan J Health Res. 2012;14(4).

40. Benbakhta B, Tazi M, Benjaafar N, Khattabi A, Maaroufi A. Determinants of patient and health system delays for women with breast cancer in Morocco, 2013. Rev Epidemiol Sante Publique. 2015;63(3):191-201. doi:10.1016/j.respe.2015.03.121

41. Joffe M, Ayeni O, Norris SA, et al. Barriers to early presentation of breast cancer among women in Soweto, South Africa. PLoS One. 2018;13(2):e0192071. doi:10.1371/journal.pone.0192071 


\section{Publish your work in this journal}

The Journal of Multidisciplinary Healthcare is an international, peerreviewed open-access journal that aims to represent and publish research in healthcare areas delivered by practitioners of different disciplines. This includes studies and reviews conducted by multidisciplinary teams as well as research which evaluates the results or conduct of such teams or healthcare processes in general. The journal covers a very wide range of areas and welcomes submissions from practitioners at all levels, from all over the world. The manuscrip management system is completely online and includes a very quick and fair peer-review system. Visit http://www.dovepress.com/testimonials. php to read real quotes from published authors. 